Journal of Inclusion Phenomena and Macrocyclic Chemistry

Review Article

\title{
Sugar-responsive smart materials based on phenylboronic acid and cyclodextrin
}

Yuya Egawa*, Tomohiro Seki, Ryotaro Miki, and Toshinobu Seki

Faculty of Pharmacy and Pharmaceutical Sciences, Josai University, 1-1 Keyakidai, Sakado, Saitama 350-0295, Japan

* yegawa@josai.ac.jp

Tel +81-49-271-7686

Fax +81-49-271-7714

\begin{abstract}
This review focuses on sugar-responsive materials based on phenylboronic acid (PBA) as a sugar-sensing motif and cyclodextrins (CyDs) as a basic skeleton of smart materials. PBA modified $\alpha$-CyD (PBA- $\alpha-C y D)$ forms a supramolecular polymer through intermolecular interactions between PBA part and CyD cavity. Similarly, PBA- $\beta$ CyD forms a head-to-head dimer. Meanwhile, combining PBA- $\gamma-C y D$ and polyethylene glycol (PEG) produces a molecular necklace. Additionally, combining PBA-modified PEG and native $\alpha$-CyD or $\gamma$-CyD results in another type of molecular necklace. These supramolecular structures are obtained as powders, and their solubility increases in the presence of sugar. Besides the powder type, a unique gel is formed through crosslinking polyvinyl alcohol (PVA) with PBA-triazole- $\gamma-\mathrm{CyD}$ (PBA-Tri- $\gamma-\mathrm{CyD}$ ). This gel can contain model drug, and it shows sugar-responsive drug release.

The sugar response of all of these smart materials can be explained by the concept of equilibrium. The smart materials are constructed with CyD-guest interactions. The CyD-guest equilibrium moves by a reaction between sugar and PBA moiety attached to the smart material. In these smart materials, sugar induces a dissociation in the CyD-guest interaction, and this dissociation results in sugar-induced disintegration of CyD-guest supramolecular structures.
\end{abstract}

\section{Keywords}

cyclodextrin; gel; phenylboronic acid; pseudopolyrotaxane; smart material; sugar response, supramolecular polymer

\section{Introduction}

Insulin is a blood sugar-lowering hormone that is widely used in the treatment of diabetes patients. There are various types of insulin formulations in the market, which have different acting times, ranging from rapid-acting and short-acting to intermediate-acting and long-acting [1]. These insulin formulations mimic the insulin secretion pattern 
of insulin by the pancreas. However, imitating pancreas insulin secretion is difficult, as evident by different kinds of formulations. In a healthy body, a small amount of insulin is secreted throughout the day [2]. After a meal, the blood glucose level increases, inducing the additional secretion of insulin to lower this level to a normal range. Thus, pancreas secretes the required amount of insulin when needed. To imitate this complicated secretion pattern, some insulin formulations are combined. However, current insulin administration patterns are not ideal, and the danger of abnormally low blood sugar remains problematic. Given these circumstances, the development of an artificial pancreas that automatically monitors glucose and accurately manages insulin delivery is one of the ultimate goals of insulin formulation $[3,4]$.

To develop an ideal insulin treatment system like an artificial pancreas, two functions are needed. The first function is a sugar-sensing capability to monitor blood glucose levels. The second one is a mechanism for releasing insulin when the blood glucose level increases. An approach that uses an electrical glucose sensor and electrical insulin pump incorporates these two functions [1]. To date, some products incorporating this approach are available in the market [5]. An alternative to using electronic devices, is to incorporate the two functions into an insulin formulation. For this alternative, one of the promising strategies is the use of smart materials - those that undergo structural changes in response to external stimuli such as light, heat, $\mathrm{pH}$, or chemical compounds [6-8]. A smart material that responds to a chemical stimulus of sugar could find application as an ideal insulin formulation. Using phenylboronic acid (PBA) as a sugar-sensing moiety and cyclodextrin (CyD) as a basic skeleton of smart materials, we have been developing sugar-responsive smart materials for an artificial pancreas [9-14].

\section{Basic intermolecular interactions used in sugar-responsive smart materials}

\section{2-1. Cyclic ester formation between PBA and sugar}

It is widely known that PBA reacts with 1,2-diol or 1,3-diol part of sugar to form a cyclic ester [15]. In the cyclic ester, the boron atom tends to be in an anionic $\mathrm{sp}^{3}$ hybridized form to accept a hydroxide ion. Accordingly, the cyclic ester formation accompanies the addition of hydroxide. This formation is fast and reversible and is followed by the reaction reaching an equilibrium state with three species: PBA, sugar, and the cyclic ester. These reactions are summarized in Equilibrium 1 in Fig. 1(a).

(a)

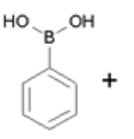

(b)

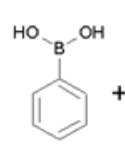

(c)

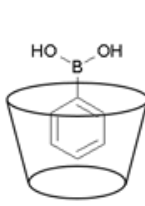

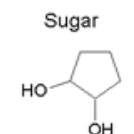

$\overline{\text { Equilibrium } 1}$
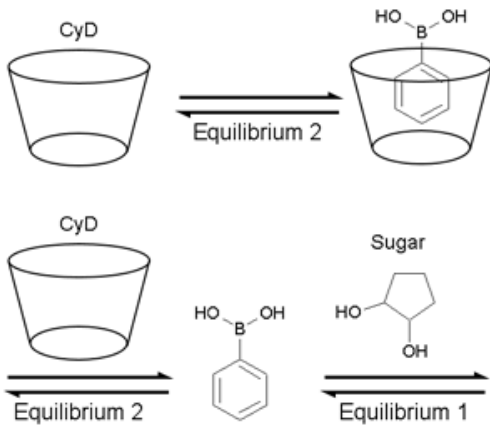

$\mathrm{HO}_{-} \mathrm{OH}$

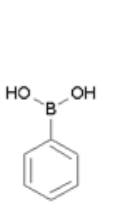

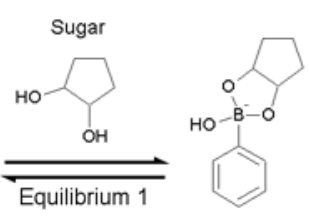


Fig. 1 Equilibriums used in some smart materials based on PBA and CyD: (a) A cyclic ester formation between PBA and sugar, (b) an inclusion complex formation between PBA and CyD, and (c) the effect of sugar on the inclusion complex of PBA and CyD.

According to the law of mass action, the binding constant $(K)$ of Equilibrium 1 reaches a fixed certain value at a given temperature. Therefore, the ratio of free PBA and the cyclic ester changes depending on initial sugar concentration. Here, we explain the concept by using a simple example.

$\mathrm{PBA}+$ Sugar $\rightleftharpoons$ Ester $\left(K=1 \mathrm{M}^{-1}\right)$

When the initial concentrations are $[\mathrm{PBA}]_{0}=[\text { Sugar }]_{0}=2 \mathrm{M}$, the concentrations of the species reach $[\mathrm{PBA}]=$ [Sugar $]=[$ Ester $]=1 \mathrm{M}$ to achieve $K=1 \mathrm{M}^{-1}$, and the ratio of [Ester]/[PBA] is 1 . If the initial concentrations change to $[\mathrm{PBA}]_{0}=2 \mathrm{M}$ and $[\text { Sugar }]_{0}=4.5 \mathrm{M}$, the concentrations of the species reach $[\mathrm{PBA}]=0.5 \mathrm{M}$, $[$ Sugar $]=3$ $\mathrm{M}$, and [Ester] $=1.5 \mathrm{M}$ to maintain $K=1 \mathrm{M}^{-1}$. In this case, the ratio of [Ester]/[PBA] increases to 3. If the ratio of [Ester]/[PBA] can be estimated with analytical instruments such as those for absorption spectrometry, fluorometry, and electrochemistry then we can calculate the initial sugar concentration [15-17]. Based on this concept, numerous sugar sensors have been developed. We also have developed boronic acid azo dyes [18] and borinate-containing xanthene derivatives named Josai-Red [19], which act as colorimetric and fluorometric sugar sensors.

This review introduces applications of sugar-sensing capabilities of PBA in sugar-responsive smart materials. Some smart materials use the equilibrium between PBA and sugar and an inclusion equilibrium between PBA and CyD.

\section{2-2. Inclusion complex formation between PBA and CyD}

CyDs are a series of oligosaccharides, which include $\alpha$-D-glucopyronose units linked by $\alpha-1,4$ glycoside bonding. The CyDs composed of 6, 7, and $8 \alpha$-D-glucopyronose units are widely used, and they are referred to as $\alpha$-CyD, $\beta$ CyD, and $\gamma$-CyD, respectively [20]. In the case of our smart materials introduced in this review, PBA and CyD do not react to form a cyclic ester. The difficulty of a cyclic ester formation between PBA and CyD can be explained by a consideration of PBA's selectivity. PBA usually binds with cis-diol of sugars [21]; however, CyDs do not contain cis-diol in their structure. Instead of reacting to form a cyclic ester, PBA and CyD interact to form an inclusion complex (Fig. 1(b)). The inclusion between PBA and $\alpha$-CyD was confirmed by our previous research in which we monitored the change in $\mathrm{p} K_{\mathrm{a}}$ of PBA [9]. The toroidal shape of CyDs enables the formation of the inclusion complex. The CyD cavity is hydrophobic inside, and this internal cavity is where the hydrophobic guest is included. The molecular form of PBA can be a guest for CyDs because of its relatively hydrophobic character [22]. Some previous reports have shown that supramolecular complexes composed of CyD and PBA derivative can act as novel sugar sensors [23-25]. In this report, the inclusion equilibrium of PBA and CyDs is important for constructing smart materials. In addition, we postulate that CyDs tend not to include sugar-bound PBA because of its bulkiness and hydrophilicity. 


\section{2-3. The effect of sugar on CyD/PBA complex}

Using equilibriums, we can explain the effect of sugar on complex PBA-CyD (Fig. 1(c)). At first, when free PBA and $\mathrm{CyD}$ are dissolved in water, they form an inclusion complex. In the equilibrium state, there are three speciesCyD, PBA and CyD/PBA inclusion complex. When sugar is added to the solution, free PBA reacts with the sugar, resulting in a decrease in the amount of free PBA (Equilibrium 1 in Fig. 1(c)). On the basis of Le Chatelier's principle, the amount of free PBA is compensated by the disintegration of CyD/PBA complex. On the basis of the movement of chemical equilibrium, sugar can trigger the disintegration of supramolecular structures. This disintegration is regarded as a response of smart materials introduced in this review.

\section{Powder-type smart materials}

\section{3-1. Supramolecular polymer based on PBA-modified $\alpha$-CyD}

If all species in Fig. 1(c) dissolve in water, it would be difficult to use them for DDS materials. To obtain smart materials, it is appropriate that only the CyD/PBA inclusion complex shows a low solubility in water and tends to exist in a solid form. In such a case, the sugar induces dissolution of the CyD/PBA complex. This sugar-induced phase change is suitable for a large response by smart materials.

We found that a chemical modification of the PBA moiety to $\alpha$-CyD was effective for obtaining a low-solubility CyD/PBA complex because unique supramolecular structures are formed through host-guest interactions between CyD and PBA moieties. They are recognized as a supramolecular structures based on guest-modified CyDs [26].

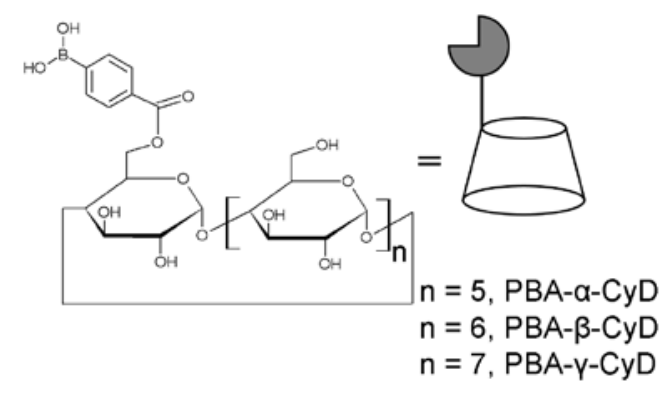

Fig. 2 Chemical structures of mono-PBA-modified CyDs.

(a)

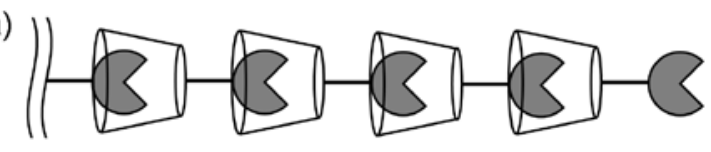

(b)

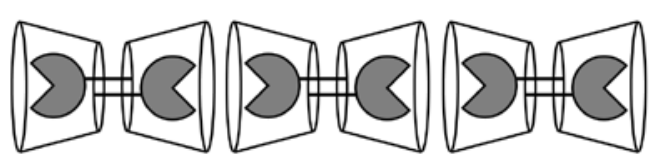

Fig. 3 Schematic illustrations of smart materials based on PBA-CyD. (a) PBA- $\alpha$-CyD supramolecular polymer, (b) PBA- $\beta-C y D$ dimer.

PBA was modified to a primary hydroxide group via a carbonyl ester bond, and mono-PBA-modified CyDs were 
purified by chromatography using polystyrene gel (Fig. 2). The solubility of PBA-modified $\alpha$-CyD (PBA- $\alpha-C y D)$ was $3.0 \mathrm{mM}$ at $37^{\circ} \mathrm{C}$ in water; a lower solubility than that of native $\alpha-\mathrm{CyD}\left(270 \mathrm{mM}\right.$ at $37^{\circ} \mathrm{C}$ in water). This low solubility was derived from a formation of a supramolecular polymer (Fig. 3(a)) [27, 28] in which host-guest interactions are repeated in a head-to-tail manner, as confirmed by two-dimensional (2D) nuclear magnetic resonance (NMR) and powder x-ray diffraction (pXRD) [9].

The supramolecular polymer based on PBA- $\alpha$-CyD shows sugar-responsive dissolution. Fig. 4(a) shows a turbidity test using an absorption spectrometer for a suspension solution containing solid-form PBA- $\alpha$-CyD. After the turbidity reached a constant value, a stock sugar solution was added to the suspended solution. Adding Dfructose (Fru) and D-glucose (Glc) induced a decrease in turbidity; thus, a sugar-responsive dissolution of the solid form PBA- $\alpha$-CyD occurred. Fru was more effective than Glc, and this selectivity is recognized as evidence for the PBA moiety acting as a sugar-sensing motif because Fru has a larger binding constant to the PBA derivative (Table 1).
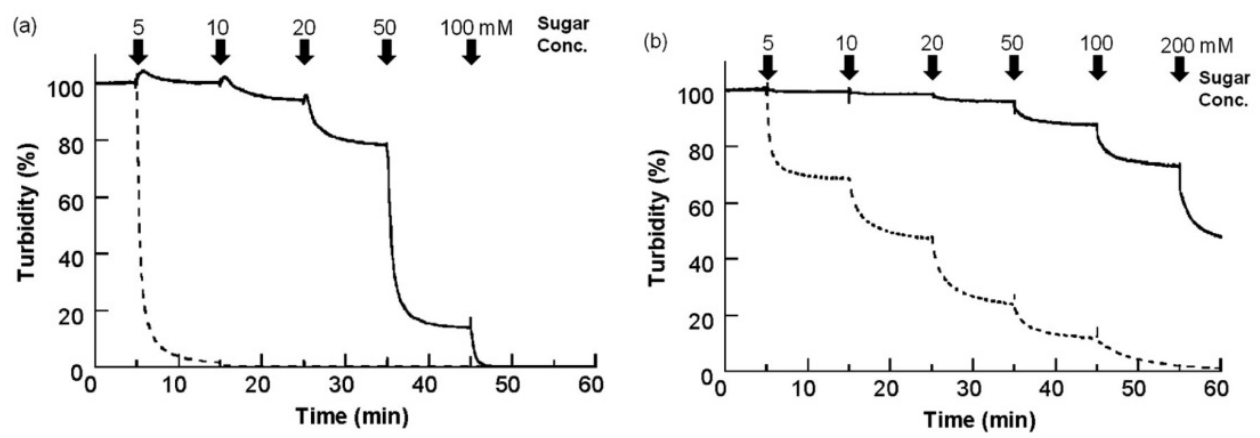

Fig. 4 Sugar-dependent turbidity changes of (a) PBA- $\alpha-C y D$ and (b) PBA- $\beta-C y D$ in PBS (pH 7.4), with the solid line indicating Glc and the dashed line Fru. Reprinted with permission from [9]. Copyright 2013, The Pharmaceutical Society of Japan.

Table 1 Binding constants $\left(\mathrm{M}^{-1}\right)$ of PBA- CyD and sugars in PBS (pH 7.4) at $37^{\circ} \mathrm{C}[9]$.

\begin{tabular}{ccc}
\hline PBA-CyD & Glc & Fru \\
\hline \hline PBA- $\alpha-C y D$ & 22 & 740 \\
PBA- $\beta-C y D$ & 29 & 720 \\
\hline
\end{tabular}

In the PBA- $\alpha$-CyD supramolecular polymer, the PBA moiety is included by the $\alpha$-CyD cavity. Here, a question arises: how does PBA react with sugar? To answer this question, we used equilibriums (Fig. 5(a)). 
(a)

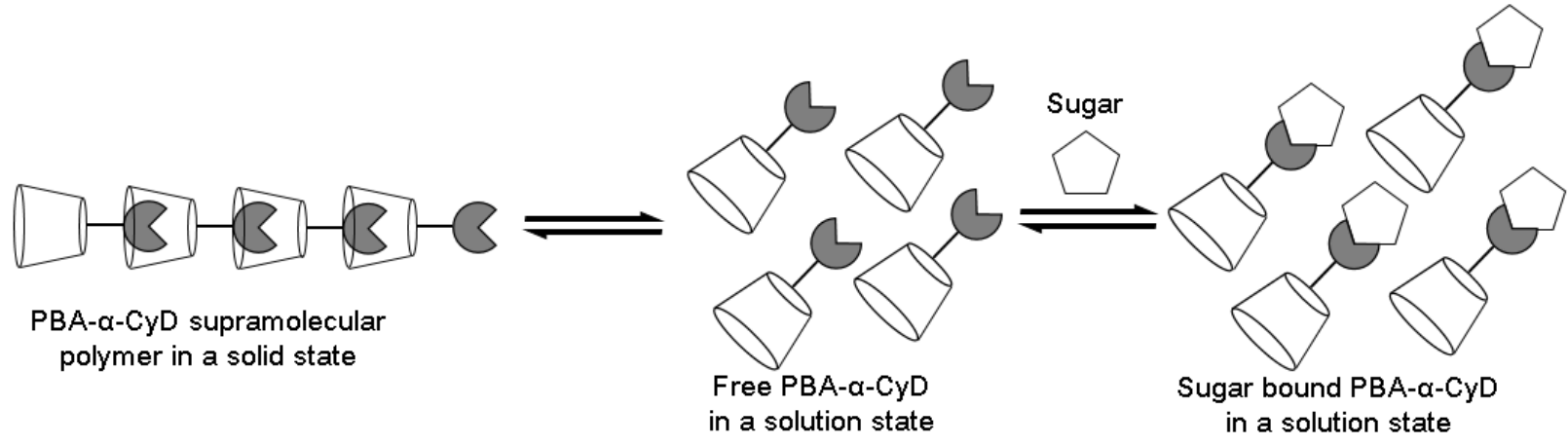

(b)

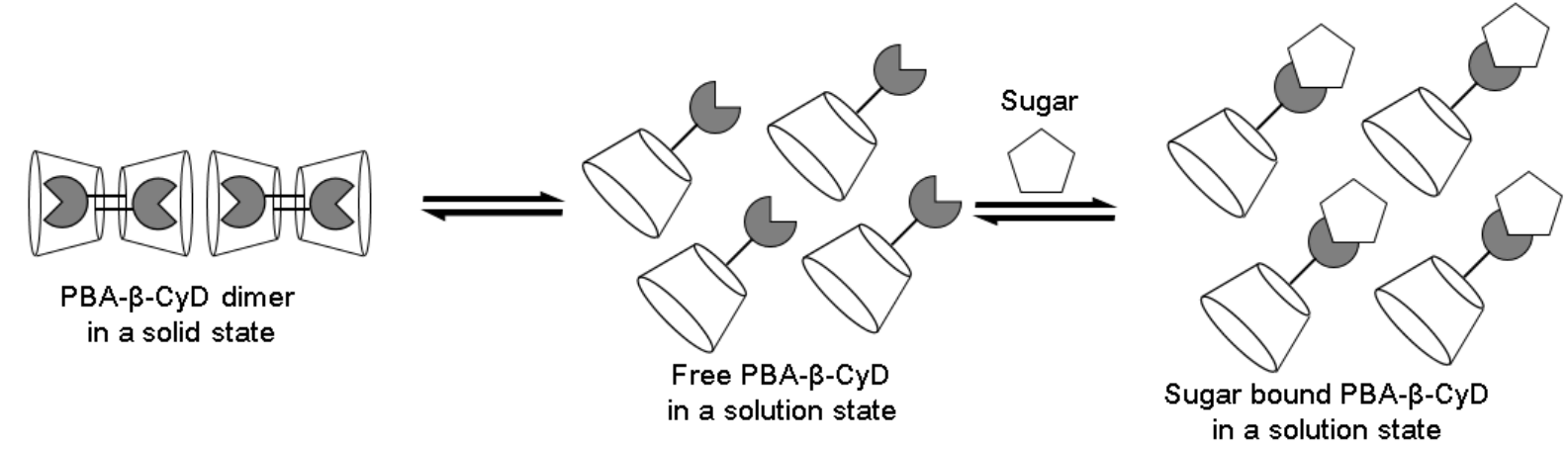

Fig. 5 The mechanism of sugar-induced disintegration for the (a) PBA- $\alpha$-CyD supramolecular polymer and the (b) PBA- $\beta-C y D$ head-to-head dimer.

In a suspension solution including PBA- $\alpha-\mathrm{CyD}$ as a solid, part of the PBA- $\alpha-\mathrm{CyD}$ dissolves in the solution. The solid state of the PBA- $\alpha$-CyD supramolecular polymer and the dissolved state of PBA- $\alpha$-CyD are in solubility equilibrium. When sugar is added to the suspension solution, the dissolved state of PBA- $\alpha-C y D$ and sugar can react to form a cyclic ester. This reaction involves a consumption of free PBA- $\alpha-C y D$ in the dissolved state. According to Le Chatelier's principle, this consumption is compensated by the movement of equilibrium. In this case, the free PBA- $\alpha-C y D$ in the dissolved state is produced by the dissociation of the solid state of the PBA- $\alpha-C y D$ supramolecular polymer. This mechanism, shown in Fig. 5(a), explains the sugar-responsive disintegration of the solid state of the PBA- $\alpha$-CyD supramolecular polymer, which is an application of the equilibriums in Fig. 1(c).

\section{3-2. Head-to-head dimer based on PBA-modified $\beta$-CyD}

Compared to $\alpha$-CyD, $\beta$-CyD has a larger ring consisting of seven $\alpha$-D-glucose units; therefore, it can deeply include the PBA moiety, which is confirmed by nuclear Overhauser enhancement and exchange spectroscopy [9]. We conducted diffusion-ordered spectroscopy of the PBA- $\beta-C y D$ solution and a pXRD pattern of PBA- $\beta-C y D$ in the solid state, and the results showed that PBA- $\beta-$ CyD forms a dimer through head-to-head interactions (Fig. 3(b)). This deep head-to-head bidentate interaction induces quite a low solubility $\left(0.23 \mathrm{mM}\right.$ in water at $\left.37^{\circ} \mathrm{C}\right)$.

Fig. 4(b) shows the sugar response of the head-to-head dimer of PBA- $\beta-C y D$, and this response is relatively small compared to that of PBA- $\alpha-C y D$. This small response is due to the low solubility of the PBA- $\beta$-CyD dimer, which forms a stable crystalline structure. Fig. 5(b) shows the sugar-response mechanism of the head-to-head dimer of PBA- $\beta-C y D$, which is basically the same as the one for the PBA- $\alpha-C y D$ supramolecular polymer. Because of 
their sugar-responsive character, the solid-state PBA- $\alpha$-CyD or PBA- $\beta-C y D$ have the potential to be base materials for sugar-responsive insulin release systems.

\section{3-3. Molecular necklace based on PBA-modified $\gamma$-CyD and PEG}

\section{(a)}

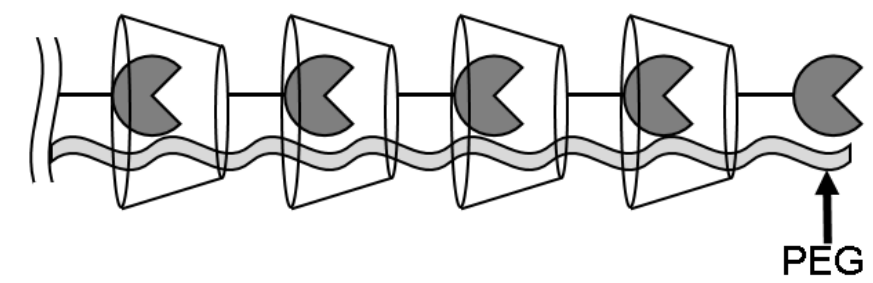

(b)

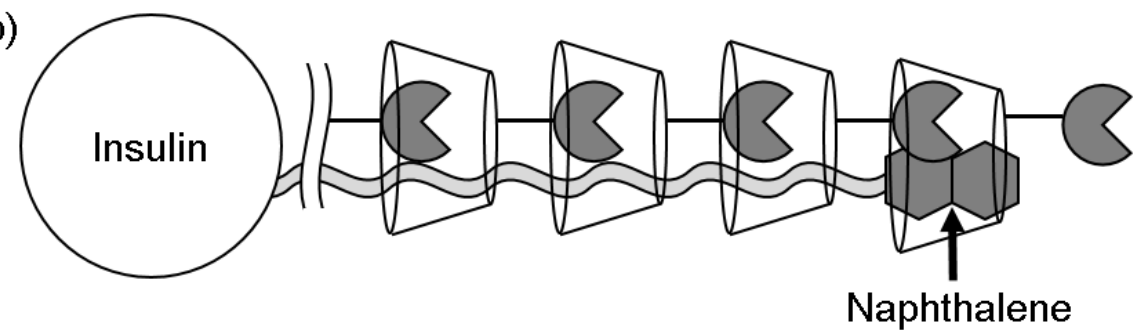

Fig. 6 Structure of molecular necklaces. (a) The molecular necklace composed of PBA- $\gamma-C y D$ and PEG. (b) The molecular necklace composed of PBA- $\gamma$-CyD and Naph-PEG-Ins.

In contrast to PBA- $\alpha-C y D$ and PBA- $\beta-C y D, P B A-\gamma-C y D$ did not show a decrease in solubility in water compared to native $\gamma$-CyD because the wide cavity of $\gamma$-CyD is composed of eight glucose units, which is too large to fit the PBA moiety. To form another type of smart material, we occupied the gap between $\gamma$-CyD and PBA with PEG (Fig. 6). The coexistence of PBA- $\gamma$-CyD and PEG in water induced a formation of a crystalline PBA- $\gamma$-CyD molecular necklace (Fig. 6(a)). The crystalline formation represents the application of a concept known as pseudopolyrotaxane, which is also referred to as a molecular necklace, and its unique structure has attracted much attention in various fields [29, 30].

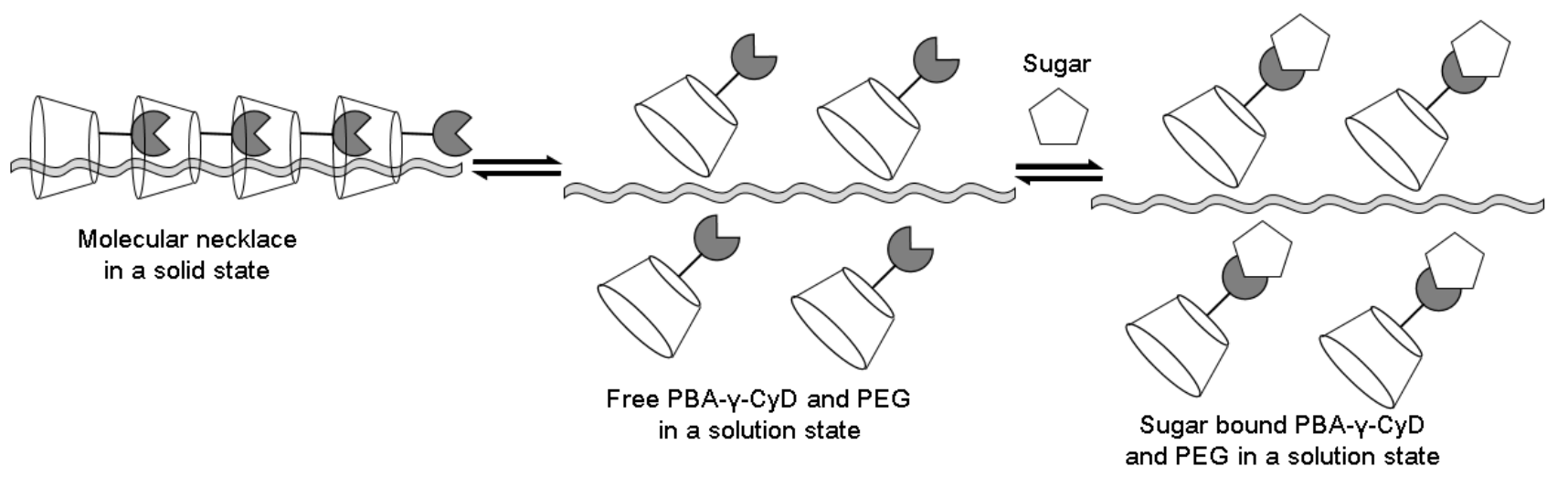

Fig. 7 The sugar-responsive mechanism of the molecular necklace composed of PBA- $\gamma$-CyD and PEG.

The molecular necklace composed of PBA- $\gamma-\mathrm{CyD}$ and PEG tends to exist in a solid state in water, and it 
disintegrates in the presence of sugar. This sugar response, illustrated in Fig. 7, relies on a similar mechanism to that of PBA- $\alpha-C y D$ and PBA- $\beta-C y D$. When the molecular necklace is added to a water solution, part of the molecular necklace remains as a solid in suspension, and another part of the molecular necklace dissolves. Then, the dissolved state of free PBA- $\gamma-C y D$ can react with sugar, decreasing the amount of free PBA- $\gamma-C y D$. To compensate the reduced amount of PBA- $\gamma-\mathrm{CyD}$, the molecular necklace disintegrates.

Notably, the PBA- $\gamma$-CyD/PEG necklace offers a new type of smart material that includes PEG-modified insulin as a component. We prepared PEG-modified insulin and combined it with PBA- $\gamma$-CyD to form a molecular necklace. We used $\left[\mathrm{H}_{2} \mathrm{~N}-\left(\mathrm{CH}_{2}\right)_{3}-\mathrm{O}-\mathrm{PEG}-\left(\mathrm{CH}_{2}\right)_{5}-\mathrm{COOH}\right]$ and modified its amine end with a naphthoyl group. The carboxylic terminal was linked to amino groups of insulin, and we referred to this conjugate as Naph-PEG-Ins (Fig. 6(b)) [10]. Naphthalene was used because it facilitates the formation of the molecular necklace. Additionally, naphthalene was useful in evaluating the release amount of insulin derivative via a fluorometric measurement.

Fig. 8 shows the release rate of the insulin derivative, which was accelerated in the presence of Glc (Fig. 8(b)). The response was improved by using 3-nitorophneylboronic acid (NPBA) instead of PBA as a modification group for $\gamma$-CyD (Fig. 8(a)) [12]. On the basis of our investigation of circular dichroism and 2D NMR, we propose that the 3-nitorophneylboronic acid moiety tends to exist in the rim of the CyD ring, and this spatial arrangement and the relatively high affinity of the 3-nitorophneylboronic acid moiety for Glc contributes to the improved sugar responsivity (Table 2). The result suggests that the system's sugar response can be tuned for physiological conditions by selecting appropriate PBA derivatives.

(a)

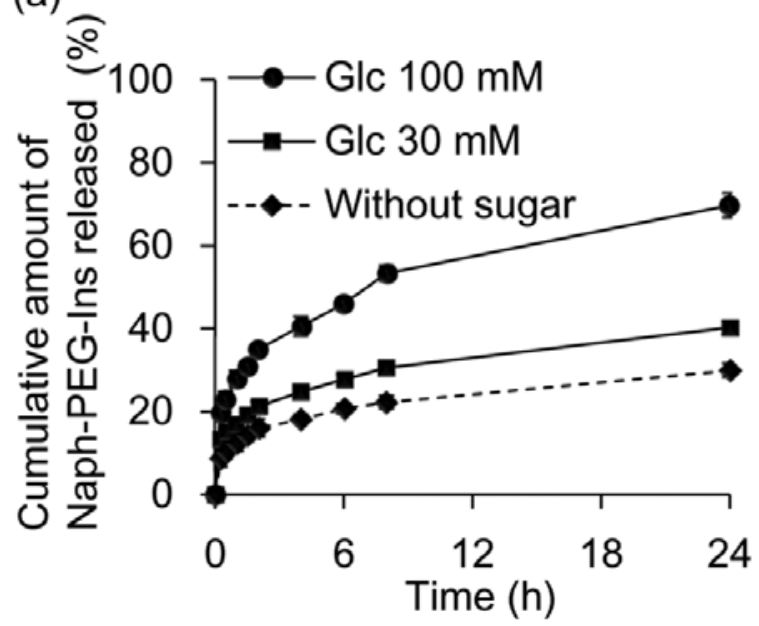

(b)

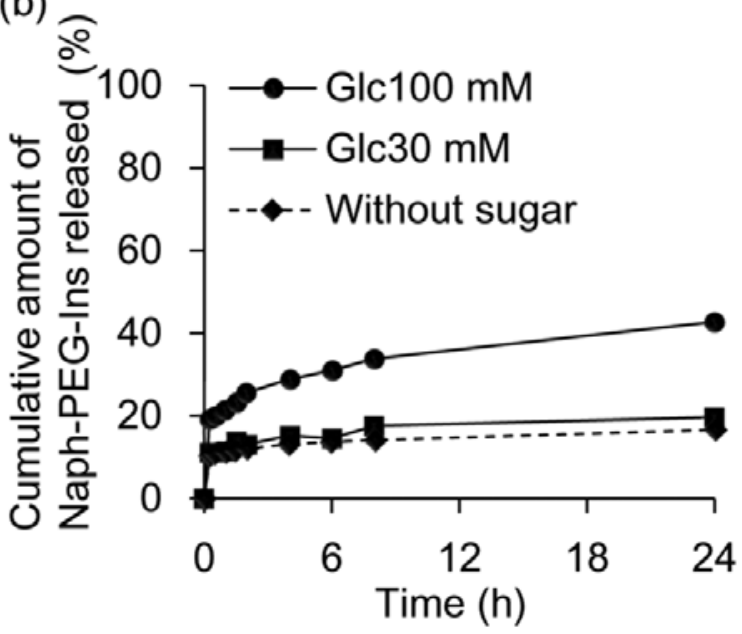

Fig. 8 Release profiles of Naph-PEG-Ins from (a) NPBA- $\gamma$-CyD/Naph-PEG-Ins molecular necklace and (b) PBA- $\gamma$ CyD/Naph-PEG-Ins molecular necklace in the absence and presence of Glc ( $\left.\mathrm{pH} 7.4,37^{\circ} \mathrm{C}\right)$. Reprinted with permission from [12]. Copyright 2016, The American Chemical Society.

Table 2 Binding constants $\left(\mathrm{M}^{-1}\right)$ of PBA- CyD and Glc in HEPES buffer (pH 7.4) at $25^{\circ} \mathrm{C}[12]$. 


\begin{tabular}{cc}
\hline PBA-CyD & Glc \\
\hline \hline PBA- $\gamma-C y D$ & 8.0 \\
NPBA- $\gamma-C y D$ & 50.1 \\
\hline
\end{tabular}

\section{3-4. Inclusion complex between native CyDs and PBA-modified PEG}

(a)

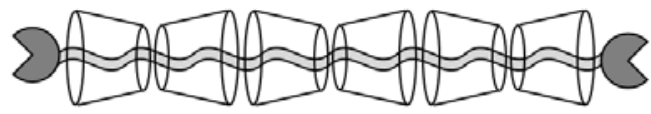

(b)

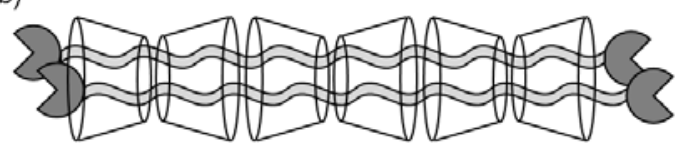

Fig. 9 Molecular necklaces composed of native CyDs and PBA-PEG. (a) A single-stranded molecular necklace consisting of $\alpha$-CyD and PBA-PEG. (b) A double-stranded molecular necklace consisting of $\gamma$-CyD and PBA-PEG.
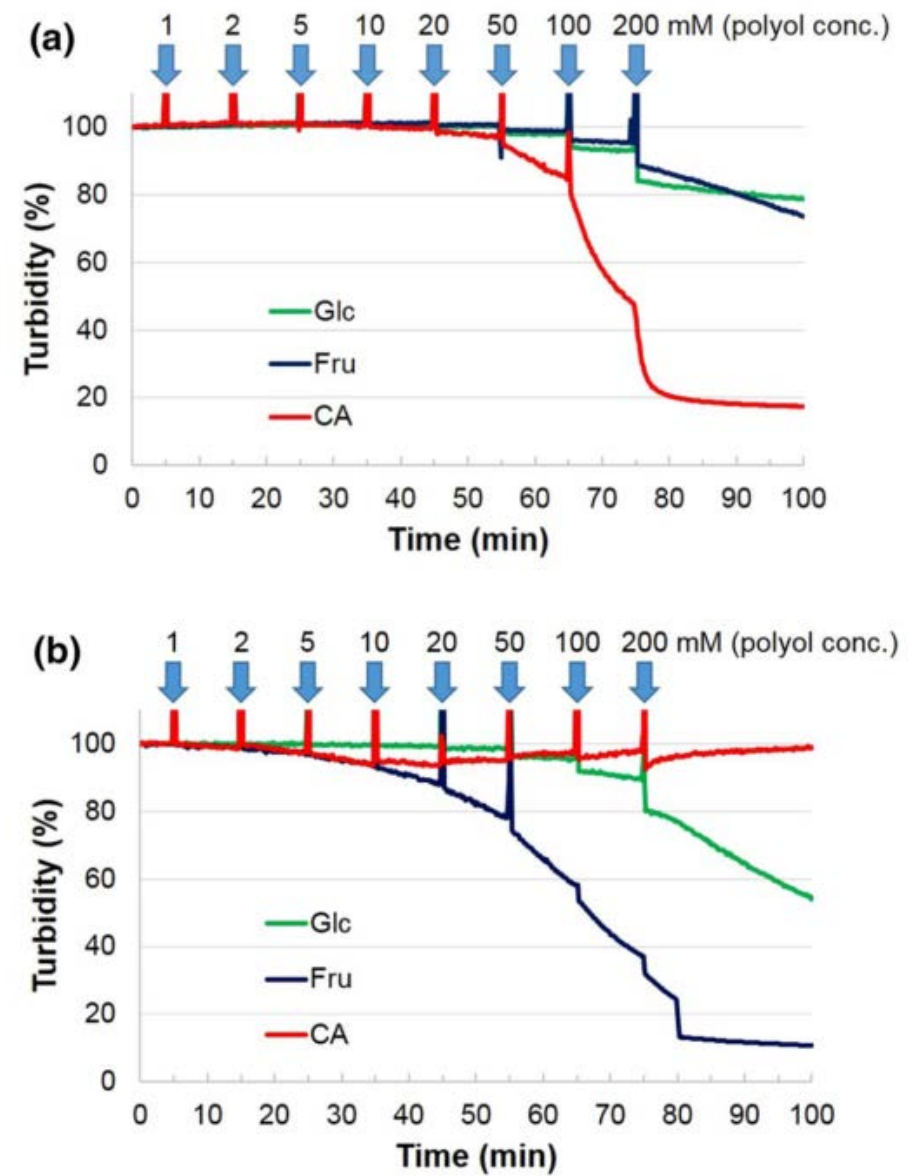

Fig. 10 Polyol responses of the molecular necklaces (pH 7.4, 37 ${ }^{\circ} \mathrm{C}$ ). (a) The molecular necklace composed of $\alpha$ CyD and PBA-PEG. (b) The molecular necklace composed of $\gamma$-CyD and PBA-PEG. Reprinted with permission from [13]. Copyright 2017, Springer. 

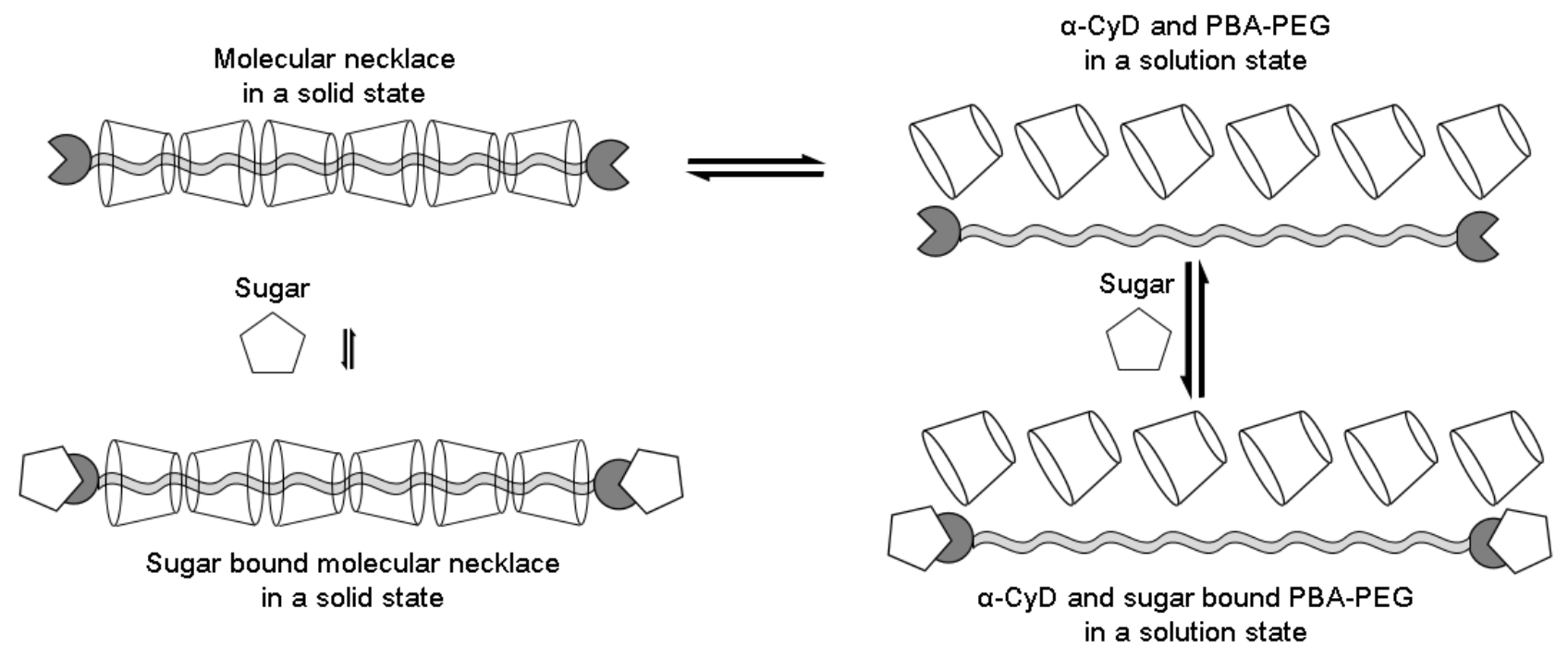

Fig. 11 The proposed sugar-response mechanism of the molecular necklace composed of $\alpha$-CyD and PBA-PEG.

In the above-mentioned cases, PBA was modified to CyDs, which are host molecules. Alternatively, we used PBA-modified guests to obtain another type of smart material. An amino-terminated PEG was used as a guest for CyDs, and the terminals of PEG were modified with 4-carboxyphenylboronic acid via amide bond, which was termed as PBA-PEG. The amide bond was suitable because $\alpha$-CyD accelerated the hydrolysis of the ester bond between the hydroxyl-terminated PEG and 4-carboxyphenylboronic acid [11]. When PBA-PEG was added to an aqueous solution of $\alpha$-CyD, a crystalline precipitate was obtained. NMR and pXRD analysis confirmed that it was a single-stranded molecular necklace, as shown in Fig. 9(a), as was the case for the molecular necklace composed of $\alpha$-CyD and PEG [29].

The effect of sugar and catechol (CA) on the necklace composed of $\alpha$-CyD and PBA-PEG was investigated using a turbidity test similar to the method previously described (Fig. 10(a)). The polyols, Glc, Fru, and CA induced a decrease in the turbidity, i.e., the solid molecular necklace disintegrated.

This response mechanism can be explained through equilibriums (Fig. 11). We assumed that the molecular necklace is only in the solid state. When the molecular necklace is suspended in an aqueous solution, part of the molecular necklace disintegrates and dissolves. In a solution, a dissolved state of PBA-PEG exists, and it can react with sugar. The sugar-bound PBA-PEG is too bulky to penetrate the cavity of CyD. Thereby, sugar acts as a blocker to form the molecular necklace, resulting in a drop in the amount of the molecular necklace. Contrastingly, when the solid-state PBA-PEG of the molecular necklace reacts with sugar, the sugar acts as a stopper to stabilize solidstate species. Thus, sugar affects the molecular necklace in two possible ways. The turbidity experiments confirmed the sugar-induced disintegration of the molecular necklace, proving that the blocker effect to prevent the formation of the molecular necklace was larger. 

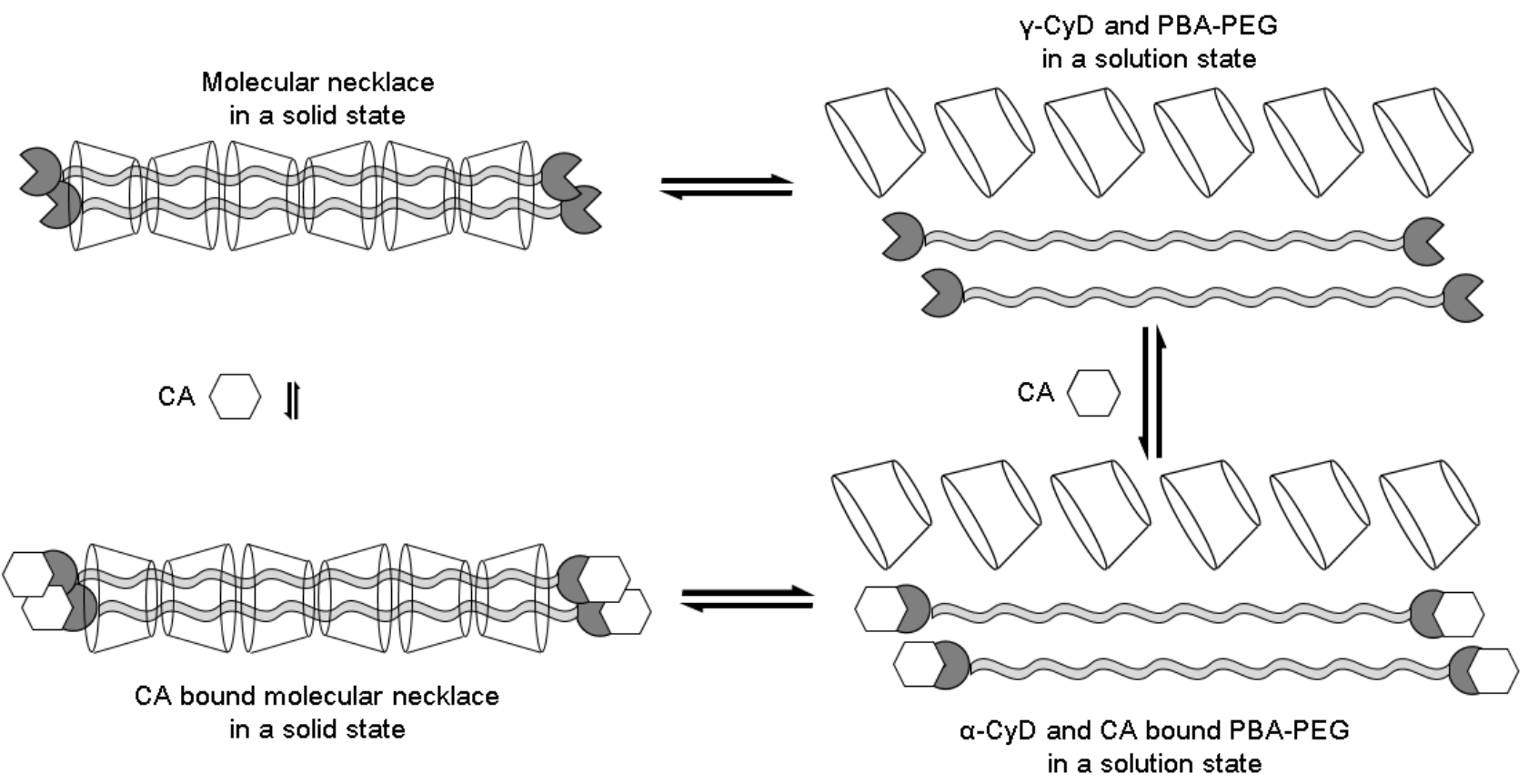

Fig. 12 A schematic of the proposed explanation for the negligible effect of CA on the molecular necklace composed of $\gamma$-CyD and PBA-PEG.

When using $\gamma$-CyD instead of $\alpha$-CyD, we obtained a double-stranded molecular necklace, (Fig. 9(b)), which was similar to that obtained for $\gamma$-CyD and modified PEG [31]. Interestingly, the double-stranded molecular necklace shows a different polyol selectivity (Fig. 10(b)) than a single-stranded molecular necklace composed of $\alpha$ CyD and PBA-PEG. The molecular necklace using $\gamma$-CyD disintegrated by Fru and Glc; did not show any response to CA.

This result can be interpreted as follows: Fru and Glc are both large enough to act as a blocker for reformation, but CA is too small. Therefore, CA does not affect the amount of the solid-state molecular necklace (Fig. 12). In other words, the $\gamma$-CyD/PBA-PEG molecular necklace possesses the selectivity of $\gamma$-CyD. This molecular necklace is an example of the combination of PBA and CyD selectivities, which will open the possibility for the development of more sophisticated smart materials.

\section{Sugar-responsive smart gel: A polyvinyl alcohol (PVA) gel crosslinked by PBA-triazole- $\gamma$-CyD (PBA-Tri- $\gamma$ - CyD)}

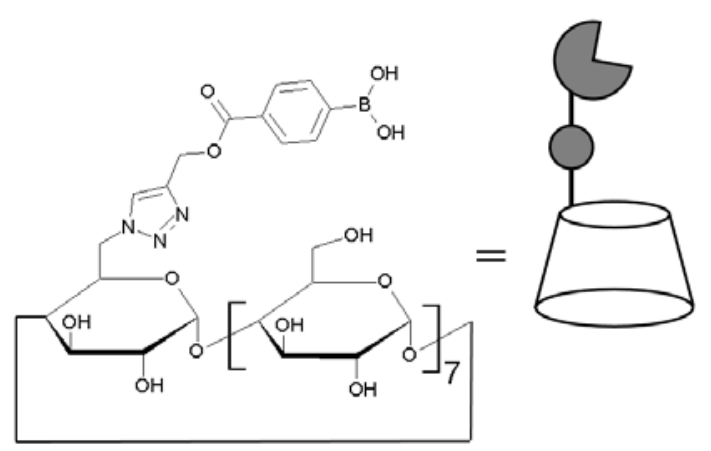


Fig. 13 Chemical structure of PBA-Tri- $\gamma$-CyD.

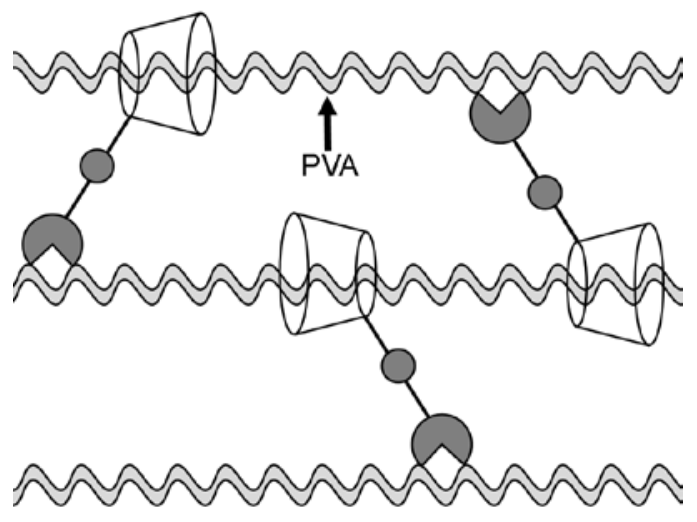

Fig. 14 Schematic illustration of a PVA-based gel crosslinked by PBA-Tri-CyD.
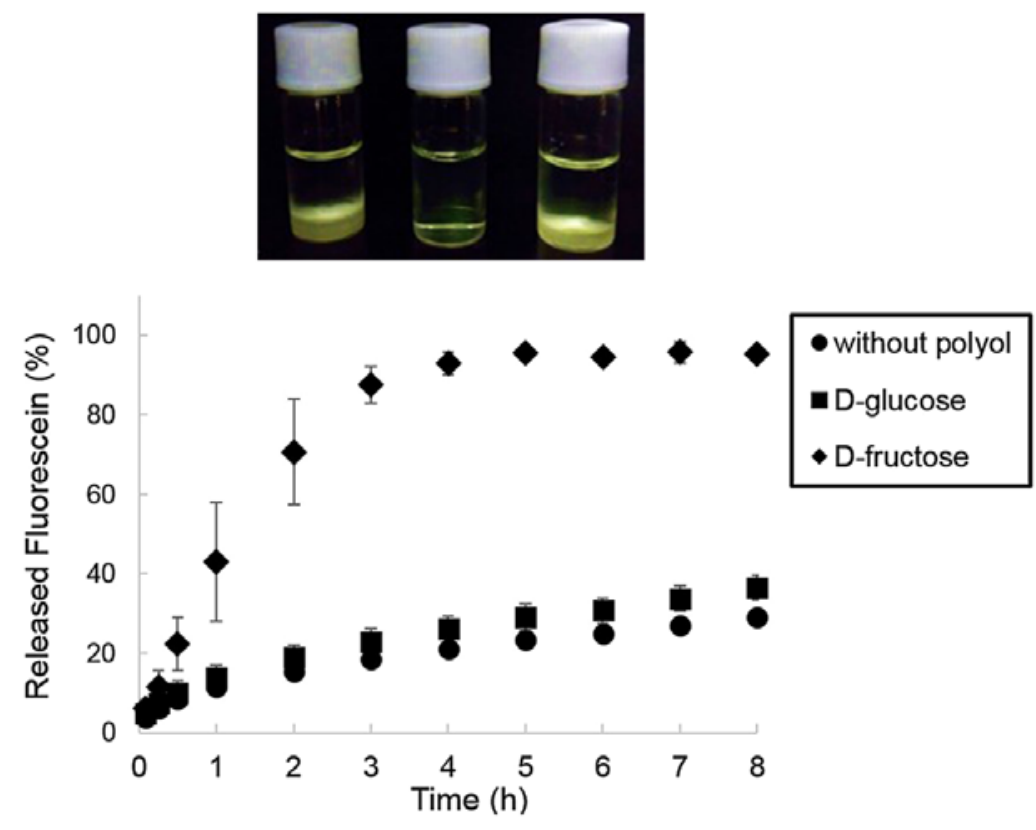

Fig. 15 Release profiles of fluorescein from a hydrogel prepared with PBA-Tri-CyD/PVA gel and the effect of polyols $\left(\mathrm{pH} 7.4,37^{\circ} \mathrm{C}, \mathrm{n}=3\right.$ ). Picture of the vial without polyol (left), with Fru (center), and Glc (right) after the release experiments. Reprinted with permission from [14]. Copyright 2017, Springer.

All of the smart materials described so far are categorized as powder type. We also attempted to develop a sugarresponsive smart gel. A gel-type formulation is attractive for sugar-responsive insulin release systems because it can incorporate insulin without chemical modification, which causes the activity loss of insulin.

For the gel preparation, PVA was chosen as a base polymer because $\gamma$-CyD includes PVA and it can interact with PBA because the repeated hydroxyl groups comprise 1,3-diols [32-38]. We prepared a PBA-appended $\gamma$-CyD via a click reaction using mono-azide $\gamma$-CyD and alkyn-modified PBA (Fig. 13). The obtained PBA-triazole- $\gamma$-CyD (PBA-Tri- $\gamma$-CyD) was used as a crosslinker for PVA (Fig. 14). The CyD moiety of PBA-Tri- $\gamma$-CyD includes a PVA chain. Simultaneously, the PBA moiety of PBA-Tri- $\gamma$-CyD forms covalent bonds with the 1,3-diol moieties of PVA. 
These two kinds of interactions—-mechanical interlocking and chemical bonding—connect PVA chains, resulting in the formation of a hydrogel.

We prepared a PBA-Tri- $\gamma$-CyD/PVA gel incorporating fluorescein as a drug molecule model and investigated the effect of sugar on the release rate of fluorescein from the gel. As shown in Fig. 15, Fru induced the acceleration of the fluorescein release, which accompanies erosion of the gel. This sugar response is based on a competitive displacement of PVA diols by sugar on the PBA moiety, resulting in a break of the crosslink.

\section{Summary and perspective}

Here, we summarized the features of smart materials whose structures balance host-guest interactions. However, the addition of sugar induces an imbalance between the host and guests because the PBA-modified host or PBA-modified guests are compensated by the binding between PBA moiety and sugar. To compensate the reduced amount, the host-guest inclusion complex disintegrates.

This is a generalized operation principle of sugar-responsive smart materials based on CyDs and PBA. However, there are several barriers to overcome before these materials can be applied in practical use for sugarresponsive insulin release systems. For example, an ideal system needs to have strict control over the insulin release rate in the range of 5 to $10 \mathrm{mM}$ Glc, which is the range of glucose level at physiological condition. Therefore, an ideal smart material would release insulin at a slow rate, similar to the rate at which basal insulin is released when the Glc is around $5 \mathrm{mM}$, and increase this rate after a meal. There is the possibility of developing glucose-selective sensors based on a PBA dimer, which works in the physiological Glc level. Some reports suggest that the PBA dimer improved the selectivity for Glc, thus, could be a promising approach for future smart materials [39, 40]. With respect to practical use, the dosage form of the smart materials must be determined. For solid-type smart materials, powder injections may be effective. For gel-type smart materials, viscosity control is needed for injections. After the injections, pharmacokinetics and sugar response should be thoroughly investigated.

We hope that this review will stimulate a disintegration of barriers existing between the fields of organic chemistry, physical chemistry, analytical chemistry, and pharmaceutics, and contribute to the formation of collaborative efforts to develop and tune smart materials for sugar-responsive drug delivery systems.

\section{References}

1. Gilroy, C.A., Luginbuhl, K.M., Chilkoti, A.: Controlled release of biologics for the treatment of type 2 diabetes. J. Control. Release. 240, 151-164 (2016). doi:10.1016/j.jconrel.2015.12.002

2. American Diabetes Association: Standards of medical care in diabetes—2017 abridged for primary care providers. Clin. Diabetes. 35, 5-26 (2017). doi:10.2337/cd16-0067

3. Sacks, D.B., Bruns, D.E., Goldstein, D.E., Maclaren, N.K., McDonald, J.M., Parrott, M.: Guidelines and recommendations for laboratory analysis in the diagnosis and management of diabetes mellitus. Clin. Chem. 48, 436-472 (2002)

4. Ravaine, V., Ancla, C., Catargi, B.: Chemically controlled closed-loop insulin delivery. J. Control. Release. 132, 2-11 (2008). doi:10.1016/j.jconrel.2008.08.009

5. Klonoff, D.C., Ahn, D., Drincic, A.: Continuous glucose monitoring: A review of the technology and clinical 
use. Diabetes Res. Clin. Pr. 133, 178-192 (2017). doi:10.1016/j.diabres.2017.08.005

6. Harada, A.: Cyclodextrin-based molecular machines. Acc. Chem. Res. 34, 456-464 (2001). doi:10.1021/ar000174l

7. Yang, Y., Hughes, R.P., Aprahamian, I.: Near-infrared light activated azo-BF2 switches. J. Am. Chem. Soc. 136, 13190-13193 (2014). doi:10.1021/ja508125n

8. Meng, F., Zhong, Z., Feijen, J.: Stimuli-responsive polymersomes for programmed drug delivery. Biomacromolecules. 10, 197-209 (2009). doi:10.1021/bm801127d

9. $\quad$ Nakamura, K., Seki, T., Egawa, Y., Miki, R., Oda, Y., Yamanoi, T., Seki, T.: Sugar-sensitive supramolecular structures based on phenylboronic acid-modified cyclodextrins. Chem. Pharm. Bull. 61, 1188-1191 (2013). doi:10.1248/cpb.c13-00542

10. Seki, T., Abe, K., Nakamura, K., Egawa, Y., Miki, R., Juni, K., Seki, T.: Sugar-responsive pseudopolyrotaxanes and their application in sugar-induced release of PEGylated insulin. J. Incl. Phenom. Macrocycl. Chem. 82, 417424 (2015). doi:10.1007/s10847-015-0504-0

11. Seki, T., Namiki, M., Egawa, Y., Miki, R., Juni, K., Seki, T.: Sugar-responsive pseudopolyrotaxane composed of phenylboronic acid-modified polyethylene glycol and $\gamma$-cyclodextrin. Materials (Basel). 8, 1341-1349 (2015). doi:10.3390/ma8031341

12. Seki, T., Abe, K., Egawa, Y., Miki, R., Juni, K., Seki, T.: A pseudopolyrotaxane for glucose-responsive insulin release: The effect of binding ability and spatial arrangement of phenylboronic acid group. Mol. Pharm. 13, 3807-3815 (2016). doi:10.1021/acs.molpharmaceut.6b00599

13. Kojima, Y., Okano, T., Seki, T., Namiki, M., Egawa, Y., Miki, R., Juni, K., Seki, T.: Polyol-responsive pseudopolyrotaxanes based on phenylboronic acid-modified polyethylene glycol and cyclodextrins. J. Incl. Phenom. Macrocycl. Chem. 87, 295-303 (2017). doi:10.1007/s10847-017-0699-3

14. Uchida, W., Yoshikawa, M., Seki, T., Miki, R., Seki, T., Fujihara, T., Ishimaru, Y., Egawa, Y.: A polyrotaxane gel using boronic acid-appended $\gamma$-cyclodextrin as a hybrid cross-linker. J. Incl. Phenom. Macrocycl. Chem. 89, 281-288 (2017). doi:10.1007/s10847-017-0755-z

15. Anzai, J.: Recent progress in electrochemical biosensors based on phenylboronic acid and derivatives. Mater. Sci. Eng. C. 67, 737-746 (2016). doi:10.1016/j.msec.2016.05.079

16. Sun, X., Zhai, W., Fossey, J.S., James, T.D.: Boronic acids for fluorescence imaging of carbohydrates. Chem. Commun. 52, 3456-3469 (2016). doi:10.1039/C5CC08633G

17. Zhang, X. tai, Liu, G. jian, Ning, Z. wei, Xing, G. wen: Boronic acid-based chemical sensors for saccharides. Carbohydr. Res. 452, 129-148 (2017). doi:10.1016/j.carres.2017.10.010

18. Egawa, Y., Miki, R., Seki, T.: Colorimetric sugar sensing using boronic acid-substituted azobenzenes. Materials (Basel). 7, 1201-1220 (2014). doi:10.3390/ma7021201

19. Shimomura, N., Egawa, Y., Miki, R., Fujihara, T., Ishimaru, Y., Seki, T.: A red fluorophore comprising a borinate-containing xanthene analogue as a polyol sensor. Org. Biomol. Chem. 14, 10031-10036 (2016). doi:10.1039/C6OB01695B

20. Jansook, P., Ogawa, N., Loftsson, T.: Cyclodextrins: structure, physicochemical properties and pharmaceutical applications. Int. J. Pharm. 535, 272-284 (2018). doi:10.1016/j.ijpharm.2017.11.018 
21. Pan, X., Yang, X., Lowe, C.R.: Evidence for a cross-linking mechanism underlying glucose-induced contraction of phenylboronate hydrogel. J. Mol. Recognit. 21, 205-209 (2008). doi:10.1002/jmr.885

22. Ozawa, R., Hayashita, T., Matsui, T., Nakayama, C., Yamauchi, A., Suzuki, I.: Effects of cyclodextrins and saccharides on dual fluorescence of N,N-dimethyl-4-aminophenylboronic acid in water. J. Incl. Phenom.

Macrocycl. Chem. 60, 253-261 (2008). doi:10.1007/s10847-007-9373-5

23. Shimpuku, C., Ozawa, R., Sasaki, A., Sato, F., Hashimoto, T., Yamauchi, A., Suzuki, I., Hayashita, T.: Selective glucose recognition by boronic acid azoprobe/ $\gamma$-cyclodextrin complexes in water. Chem. Commun. 1709-1711 (2009). doi:10.1039/b819938h

24. Kumai, M., Kozuka, S., Samizo, M., Hashimoto, T., Suzuki, I., Hayashita, T.: Glucose recognition by a supramolecular complex of boronic acid fluorophore with boronic acid-modified cyclodextrin in water. Anal. Sci. 28, 121-121 (2012). doi:10.2116/analsci.28.121

25. Tsuchido, Y., Fujiwara, S., Hashimoto, T., Hayashita, T.: Development of supramolecular saccharide sensors based on cyclodextrin complexes and self-assembling systems. Chem. Pharm. Bull. 65, 318-325 (2017). doi:doi:10.1248/cpb.c16-00963

26. Arima, H., Motoyama, K., Higashi, T.: Potential use of cyclodextrins as drug carriers and active pharmaceutical ingredients. Chem. Pharm. Bull. 65, 341-348 (2017). doi:10.1248/cpb.c16-00779

27. Hanessian, S., Benalil, A., Simard, M., Bélanger-Gariépy, F.: Crystal structures and molecular conformations of mono-6-azido-6-deoxy $\alpha$-Cyclodextrin and mono-2-O-allyl- $\alpha$-cyclodextrin -The formation of polymeric helical inclusion complexes. Tetrahedron. 51, 10149-10158 (1995). doi:10.1016/0040-4020(95)00609-C

28. Harada, A., Kawaguchi, Y., Hoshino, T.: Supramolecular polymers formed by modified cyclodextrins. J. Incl. Phenom. 41, 115-121 (2001). doi:10.1023/A:1014450521131

29. Harada, A., Li, J., Kamachi, M.: The molecular necklace: A rotaxane containing many threaded $\alpha$-cyclodextrins. Nature. 356, 325-327 (1992). doi:10.1038/356325a0

30. Wenz, G., Han, B.H., Müller, A.: Cyclodextrin rotaxanes and polyrotaxanes. Chem. Rev. 106, $782-817$ (2006). doi:10.1021/cr970027+

31. Harada, A., Li, J., Kamachi, M.: Double-stranded inclusion complexes of cyclodextrin threaded on poly(ethylene glycol). Nature. 370, 126-128 (1994). doi:10.1038/370126a0

32. Piest, M., Zhang, X., Trinidad, J., Engbersen, J.F.J.: pH-responsive, dynamically restructuring hydrogels formed by reversible crosslinking of PVA with phenylboronic acid functionalised PPO-PEO-PPO spacers (Jeffamines®). Soft Matter. 7, 11111-11118 (2011). doi:10.1039/c1sm06230a

33. Nishiyabu, R., Kobayashi, H., Kubo, Y.: Dansyl-containing boronate hydrogel film as fluorescent chemosensor of copper ions in water. RSC Adv. 2, 6555-6561 (2012). doi:10.1039/C8CC02610F

34. Nishiyabu, R., Ushikubo, S., Kamiya, Y., Kubo, Y.: A boronate hydrogel film containing organized twocomponent dyes as a multicolor fluorescent sensor for heavy metal ions in water. J. Mater. Chem. A. 2, 1584615852 (2014). doi:10.1039/c4ta03268c

35. Meng, H., Zheng, J., Wen, X., Cai, Z., Zhang, J., Chen, T.: pH- and sugar-induced shape memory hydrogel based on reversible phenylboronic acid-diol ester bonds. Macromol. Rapid Commun. 36, 533-537 (2015)

36. Duncan, T.T., Berrie, B.H., Weiss, R.G.: Soft, peelable organogels from partially hydrolyzed poly(vinyl acetate) 
and benzene-1,4-diboronic acid: Applications to clean works of art. ACS Appl. Mater. Interfaces. 9, 2806928078 (2017). doi:10.1021/acsami.7b09473

37. Peters, G.M., Chi, X., Brockman, C., Sessler, J.L.: Polyvinyl alcohol-boronate gel for sodium hydroxide extraction. Chem. Commun. 54, 5407-5409 (2018). doi:10.1039/C8CC02610F

38. Duncan, T.T., Weiss, R.G.: Influence of length and structure of aryl boronic acid crosslinkers on organogels with partially hydrolyzed poly (vinyl acetate). Colloid Polym. Sci. 296, 1047-1056 (2018)

39. Gamsey, S., Miller, A., Olmstead, M.M., Beavers, C.M., Hirayama, L.C., Pradhan, S., Wessling, R.A., Singaram, B.: Boronic acid-based bipyridinium salts as tunable receptors for monosaccharides and $\alpha$-hydroxycarboxylates. J. Am. Chem. Soc. 129, 1278-1286 (2007). doi:10.1021/ja066567i

40. Phillips, M.D., Fyles, T.M., Barwell, N.P., James, T.D.: Carbohydrate sensing using a fluorescent molecular tweezer. Chem. Commun. 6557-6559 (2009). doi:10.1039/b909230g 